Revue internationale P.M.E.

Économie et gestion de la petite et moyenne entreprise

Revuse

internationale

PME

\title{
L'accès des PME à la recherche fondamentale
}

\section{Jacqueline Senker}

Volume 7, numéro 3-4, 1994

URI : https://id.erudit.org/iderudit/1008426ar

DOI : https://doi.org/10.7202/1008426ar

Aller au sommaire du numéro

\section{Éditeur(s)}

Presses de l’Université du Québec

\section{ISSN}

0776-5436 (imprimé)

1918-9699 (numérique)

Découvrir la revue

Citer cet article

Senker, J. (1994). L'accès des PME à la recherche fondamentale. Revue internationale P.M.E., 7(3-4), 121-146. https://doi.org/10.7202/1008426ar

\section{Résumé de l'article}

Les projets de nombreux gouvernements pour promouvoir l'innovation industrielle à rintérieur des PME ont pour objectif d'encourager les relations de ces dernières avec la recherche fondamentale. Le manque de chercheurs et d'ingénieurs qualifiés constitue un des freins majeurs aux relations des PME avec la recherche fondamentale. Par contre, des études de cas sectorielles montrent que la plupart des PME souhaitant utiliser cette ressource peuvent habituellement le faire. Les cas étudiés montrent aussi que les besoins de PME en recherche fondamentale et le type de connaissances requises sont variés. Les politiques soutenant les relations des PME avec la recherche du secteur public devraient tenir compte de ces constats. En effet, il se peut que le choix réussi de certains plans spécifiques soit en relation avec des exemples de projets qui coïncident fortuitement avec les besoins d'un secteur donné. L'absence, dans des domaines particuliers, de la recherche scientifique publique est un autre frein significatif aux relations universités- entreprises. 


\title{
L'accès des PME à la recherche fondamentale
}

Jacqueline SENKER*

SPRU Université de Sussex

MOTS CLÉS

Innovation - Transfert technologique - Secteurs traditionnels Secteurs de haute technologie

\begin{abstract}
RÉSUMÉ
Les projets de nombreux gouvernements pour promouvoir l'innovation industrielle à l'intérieur des PME ont pour objectif d'encourager les relations de ces dernières avec la recherche fondamentale. Le manque de chercheurs et d'ingénieurs qualifiés constitue un des freins majeurs aux relations des PME avec la recherche fondamentale. Par contre, des études de cas sectorielles montrent que la plupart des PME souhaitant utiliser cette ressource peuvent habituellement le faire. Les cas étudiés montrent aussi que les besoins de PME en recherche fondamentale et le type de connaissances requises sont variés. Les politiques soutenant les relations des PME avec la recherche du secteur public devraient tenir compte de ces constats. En effet, il se peut que le choix réussi de certains plans spécifiques soit en relation avec des exemples de projets qui coïncident fortuitement avec les besoins d'un secteur donné. L'absence, dans des domaines particuliers, de la recherche scientifique publique est un autre frein significatif aux relations universitésentreprises.
\end{abstract}

* Jacqueline Senker est chercheuse au Science Policy Research Unit (SPRU) de l'Université de Sussex. L'auteure remercie de son soutien le service innovation du ministère du Commerce et de l'Industrie (DTI, Department of Trade and Industry), pour la préparation d'un compte rendu à partir duquel cet article a été rédigé. Les idées exprimées sont uniquement celles de l'auteure et ne reflètent pas nécessairement celles du ministère du Commerce et de l'Industrie. La traduction a été réalisée par A. Barek et L. Fragnaud du DESS Évaluation et maîtrise de l'innovation et de la technologie de l'Université Lyon 2, sous la direction de Christian Le Bas (LESA). Adresse: Science Policy Research Unit, University of Sussex, Falmer, Brighton BN1 9RF, Angleterre. 


\begin{abstract}
Many government schemes to promote industrial innovation in small and medium-sized firms (SMEs) are directed at encouraging their links with the science base. Case studies show that a major barrier to such linkage is low employment of qualified scientists and engineers in SMEs. They also show that SMEs vary in their need to access the science base, and in the type of knowledge required. Policies devised to support SMEs links with public sector research should take these variations into account.
\end{abstract}

\title{
RESUMEN
}

Muchos esquemas gubernamentales de apoyo a la innovacion industrial en pequenas y medianas empresas (PyMEs) se dirigen a promover su engranaje con la base cientifica del pais. Estudios de caso muestran que tal fin se ve dificultado por el bajo emplio de cientificos y ingenieros cualificados en las PyMEs. También muestran las diferencias que existen entre las mismas PyMEs por lo que se refiere a las necesidades de acceso a la base cientifica y al tipo de conocimientos requeridos. Las politicas de apoyo al estrechamiento de los lazos entre PyMEs e investigacion en el sector publico deben tomar en cuenta tales diferencias.

\section{Introduction}

Cet article étudie l'accès des PME à la recherche fondamentale. La recherche fondamentale est définie comme l'accumulation de connaissances, de méthodes, de talents et de matériels scientifiques obtenus au sein de l'organisation de la recherche du secteur public: universités, instituts et associations de recherche. La première partie de cet article analyse la littérature générale récente traitant du rôle des PME dans l'innovation. Plus précisément, nous identifierons les PME qui sont supposées bénéficier de l'accès à la recherche fondamentale. La seconde partie de cet article traite de la recherche fondamentale, des diverses politiques destinées à promouvoir les relations entre les industries et la recherche universitaire. On y examine aussi les facteurs qui gênent une telle relation. La troisième partie présente en détails des cas récents de coopération, dans divers secteurs, de PME avec la recherche fondamentale. Cette approche sectorielle diffère des nombreuses études qui utilisent des critères de localisation ou les entreprises de haute technologie comme base de l'échantillon.

\section{PME et innovation}

La capacité des sociétés à transformer des idées en produits et procédés couronnés de succès au niveau commercial, c'est-à-dire l'innovation, est au centre de la croissance économique. La base de données SPRU, recensant 
plus de 4000 innovations industrielles significatives en Grande-Bretagne au cours de la période 1949-1983, montre la relative contribution des PME à l'innovation, ainsi que les idées créatrices utilisées dans ces innovations. Pendant cette période, les petites et grosses entreprises ont augmenté leur part relative d'innovation, avec cependant une baisse dans la part des moyennes entreprises, mais pas uniformément dans tous les secteurs. Les PME affichent une part croissante dans les innovations des machines, des outils, des véhicules, du bâtiment et du forage.

Dans le tableau 1, une analyse plus approfondie sur plus de 2000 de ces innovations montre que, durant la période 1970-1979, les petites sociétés (employant moins de 200 personnes) ont presque doublé leur pourcentage d'innovations et d'idées nouvelles provenant de sources externes par rapport à la période précédente. Durant la même période, la part des petites entreprises a atteint $19,5 \%$ de la totalité des innovations. Les données collectées pour la période 1980-1983 montrent une augmentation supplémentaire de la part d'innovations des petites firmes pour atteindre $26,3 \%$. Ces données révèlent que depuis 1970 , les petites sociétés du Royaume-Uni utilisent plus de connaissances externes et augmentent ainsi leur contribution à l'innovation nationale.

TABleau 1

Principales sources de connaissances utilisées

dans 220 innovations importantes introduites au Royaume-Uni entre 1945 et 1979

\begin{tabular}{l|c|c|c|c}
\hline & \multicolumn{2}{|c|}{$1945-1969$} & \multicolumn{2}{c}{$1970-1979$} \\
\hline & \multicolumn{2}{|c|}{ Taille des entreprises } & \multicolumn{2}{c}{ Taille des entreprises } \\
\hline & $<200$ & $>10000$ & $<200$ & $>10000$ \\
\hline Interne & $83 \%$ & $58 \%$ & $68 \%$ & $77 \%$ \\
\hline Externe & $17 \%$ & $43 \%$ & $32 \%$ & $23 \%$ \\
\hline
\end{tabular}

Source : R. Rothwell et M. Beesley, «The Importance of Technology Transfer», dans J. Barber, S. Metcalfe et M. Porteous (dir.), Barriers to Growth in Small Firms, Londres, Routledge, 1989.

Une enquête menée à partir d'un questionnaire sur les PME issues des bases de données du SPRU montrait qu'elles entretenaient de nombreuses relations externes, y compris pour des contrats et des partenariats de R-D, avec les établissements scolaires, ainsi qu'avec d'autres services du secteur public et des associations de recherche. D'autres liens furent établis par l'intermédiaire d'accords industriels et d'accords en marketing. 
La plupart de ces relations se font entre entreprises. Par exemple, $39 \%$ des entreprises sous-traitent leur R-D, principalement auprès des fournisseurs et des clients. Le principal objectif de ces contrats externes en R-D est de raccourcir les délais d'exécution (pour la moitié des cas); la raison pour un tiers des sociétés est d'avoir accès à des technologies nouvelles. Si $53 \%$ des entreprises sous-traitant leur R-D ont agi ainsi en raison d'un manque de compétences, $26 \%$ des entreprises enquêtées se sont de plus engagées dans diverses formes de partenariat en R-D, à nouveau avec les fournisseurs et les clients principalement.

Parmi les entreprises étudiées, $55 \%$ ont des contrats réguliers avec la recherche du secteur public et $51 \%$ ont utilisé des données provenant de cette source dans leurs activités innovantes ; $31 \%$ parrainent des étudiants dans leurs études et $39 \%$ fournissent des stages aux étudiants en formation professionnelle alternée. Le parrainage et les stages sont des moyens courants pour rester proche des activités de recherche menées par le secteur public.

Ce serait cependant une erreur de supposer qu'il y ait un modèle de partenariat externe dans l'ensemble des PME. Comme Rothwell l'a fait remarquer, le secteur des PME est extrêmement hétérogène, et les besoins en nouvelles connaissances techniques externes varient considérablement. Il distingue quatre grandes catégories d'entreprises:

1. Les sous-traitants qui produisent des composants et les assemblent pour répondre aux exigences précises des clients. Nombreux de ces sous-traitants ne font pas de R-D formelle et puisent la plupart de leurs connaissances technologiques auprès de leurs clients.

2. Les PME des secteurs traditionnels tels le textile, les vêtements et la métallurgie. Elles sont supposées utiliser les technologies produites par leurs fournisseurs d'intrants et d'équipements.

3. Les petites PME modernes ayant une stratégie de créneaux, par exemple, dans les instruments scientifiques et les machines spécialisées, fournissent des procédés innovateurs. Elles ont tendance à pratiquer la R-D, mais elles ont aussi besoin de données théoriques externes pour compléter leur propre capacité.

4. Les PME basées sur de nouvelles technologies et impliquées dans les technologies émergentes comme les biotechnologies, des technologies de pointe en information et les nouveaux matériaux. Ces entreprises font de la recherche intensive et, tout en accumulant des technologies internes, complètent leurs ressources par des compétences venant des universités, d'associations de recherche, des laboratoires publics et d'autres entreprises. 


\section{Les relations avec la recherche fondamentale}

La recherche fondamentale externe comprend les scientifiques technologues dirigeant la recherche au sein d'écoles, les associations de recherche coopérative, les établissements de recherche gouvernementale et les organisations indépendantes. Les informations scientifiques et techniques provenant de ces sources peuvent jouer un rôle doublement important: engendrer des idées débouchant sur l'innovation et résoudre des problèmes survenant dans les entreprises au cours d'un processus d'innovation. Les résultats de toutes ces recherches sont diffusés dans la documentation scientifique et lors de conférences.

Une récente étude fut réalisée au Royaume-Uni sur plus de 2000 PME interrogées sur l'utilisation faite des informations techniques externes et leurs types de supports : $60 \%$ de ces entreprises utilisaient ce type d'information. Comme le montre le tableau 2, les journaux professionnels et commerciaux, ainsi que les fournisseurs et les clients, représentent de loin les sources les plus importantes. Les établissements publics de recherche et les universités sont considérés comme les moins importants.

TABleau 2

Les informations techniques provenant de sources externes, utilisées par les PME

\begin{tabular}{l|c|c|c}
\hline \multicolumn{1}{c|}{ Sources externes } & $\begin{array}{c}\text { Locales } \\
\mathbf{\%}\end{array}$ & $\begin{array}{c}\text { Nationales } \\
\mathbf{\%}\end{array}$ & $\begin{array}{c}\text { Internationales } \\
\mathbf{\%}\end{array}$ \\
\hline Universités/enseignement supérieur & 12,5 & 12,2 & 3,3 \\
Institutions ou consultants & & 22,4 & 5,6 \\
de recherche privée & 2,4 & 11,6 & 2,6 \\
Établissements publics de recherche & 22,2 & 54,3 & 23 \\
Offreurs ou consommateurs & 10,7 & 23,9 & 9,6 \\
Autres entreprises & 12,4 & 59,1 & 24 \\
Revues professionnelles & $\mathbf{1 1 7 2}$ & $\mathbf{1 1 7 2}$ & $\mathbf{1 1 7 2}$ \\
\hline ou commerciales & \multicolumn{1}{c}{ Total } & & \\
\hline
\end{tabular}

Source : The State of British Enterprise, Small Business Research Centre,

Département d'économie appliquée, Université de Cambridge, 1992, p. 65. 


\subsection{Les mécanismes pour lier la recherche fondamentale et l'industrie}

Les principales activités des institutions d'enseignement supérieur sont l'enseignement pour les bac +3 et plus, et les recherches entreprises pour accroître la connaissance. Transférer cette connaissance et ces compétences aux industries se fait de façon traditionnelle par l'intermédiaire des stages d'étudiants diplômés (de trois années d'études supérieures) et ceux qui ont prolongé leurs études, et qui sont employés par la suite dans les entreprises. Ce transfert se fait aussi par l'intermédiaire de publications universitaires en ce qui concerne les résultats de recherche. Les universités peuvent aussi transférer des connaissances de différentes manières :

- en encourageant le personnel à fournir des conseils en plus des conseils d'experts professionnels;

- en créant des sociétés pour exploiter la recherche ou les activités de développement et de design des personnels d'universités.

Pendant les années 80 , il y a eu une augmentation considérable des mécanismes liant les universités et les entreprises, due à plusieurs facteurs dont une baisse du soutien aux universités de la part du gouvernement et une impossibilité à maintenir les salaires universitaires, dans l'ensemble du secteur public, comparables à ceux du secteur privé. La différence a surtout été marquante pour ceux qui possédaient des compétences en informatique et en électronique. La seule façon pour les universités de retenir leurs personnels était de les autoriser à jouer un rôle de consultant. Les changements dans la législation, qui permettaient d'exploiter des résultats de recherche publique, ont aussi eu un effet. Après 1984, les droits d'exploitation de ces résultats furent retirés au Conseil national de recherche et développement et aux conseils de recherche, pour être transférés aux universités admissibles à des subventions.

La réaction de chaque université à ces défis a considérablement varié. Leurs réactions peuvent être classées en quatre catégories. Premièrement, il y a eu des initiatives pour faciliter la communication entre les universités et l'industrie en général, notamment la promotion des expertises et moyens universitaires. Deuxièmement, les universités ont désigné des responsables de leurs liaisons avec les industries. Ces responsables ont une variété d'obligations dont la mise en relation des besoins en recherche des industriels avec le personnel universitaire en question et une assistance professionnelle à chaque université. Troisièmement, quelques universités ont créé des sociétés pour vendre leurs services ou établir des centres de recherche spécialisée. Quatrièmement, avec le soutien du gouvernement local ou des fonds commu- 
nautaires, des établissements d'enseignement supérieur se sont associés pour créer des centres de transfert de technologie afin de commercialiser la technologie de leurs établissements.

Les agences publiques et les centres de recherche ont ainsi été actifs dans le développement de projets pour promouvoir des relations universitésindustries. Le ministère du Commerce et de l'Industrie et le Centre de recherche en sciences et en technologies (SERC, Science and Engineering Research Council) ont été les plus actifs à cet égard, et se sont associés pour commanditer certains projets. Les programmes de R-D de la Communauté européenne pour la recherche, en collaboration entre les organisations appartenant à plusieurs États membres, englobent également les opportunités de collaboration entre industriels et universitaires. Le ministère du Commerce et de l'Industrie, ainsi que d'autres ministères, jouent le rôle d'agents de la Communauté européenne dans la promotion de ces projets au sein du RoyaumeUni. Le ministère du Commerce et de l'Industrie a de nombreux projets pour promouvoir l'innovation des entreprises, avec une gamme spécialement prévue pour les PME. En plus de soutenir une aide en audit et de fournir des services de transferts de technologies, divers projets offrent des subventions de collaboration avec d'autres entreprises ou universités de haut calibre. De plus, quatre agences de recherche principales du ministère du Commerce et de l'Industrie fournissent des services scientifiques et techniques payants.

La politique du SERC destinée à augmenter le soutien à la recherche à caractère industriel a évolué lentement depuis le milieu des années 70 . Elle offre aux entreprises la chance de consolider la moitié des dépenses d'un projet spécifique de recherche dans une université, avec le SERC fournissant le solde des dépenses. Elle participe aussi aux CASE («Co-operative Awards in Science and Engineering»: prix en science et ingénierie) pour les thésards afin qu'ils mènent à terme un programme de recherche conçu et supervisé à la fois par un département d'université et une entreprise. Le programme des entreprises enseignantes (Teaching Company Scheme) qui fut originellement établi par le SERC finance l'affectation provisoire de diplômés dans l'industrie dans le but de mettre en œuvre des projets spécifiques dans le domaine des affaires. Les diplômés transfèrent la nouvelle technologie à l'entreprise, en travaillant sous le contrôle d'un expert universitaire. Le projet est maintenant largement soutenu par le SERC et le ministère du Commerce et de l'Industrie, mais d'autres conseils en recherche et services sont aussi impliqués.

Le ministère du Commerce et de l'Industrie et le SERC sont aussi les principaux participants au projet LINK qui touche des domaines dans lesquels on considère que le Royaume-Uni aurait besoin de dynamiser les 
connaissances de base. Les industries contribuent à $50 \%$ dans les dépenses de recherche engagées par les universités; l'autre moitié est pris en charge par un ministère et un conseil en recherche.

\subsection{Les barrières à l'entrée de la recherche fondamentale}

Les entreprises diffèrent dans leur façon d'accéder à la recherche fondamentale ou d'utiliser ses mécanismes. Les diverses enquêtes révèlent que la tendance naturelle d'une entreprise et sa possibilité d'accéder à une recherche fondamentale externe dépendent de ses ressources techniques internes, et en particulier de l'embauche de scientifiques et d'ingénieurs qualifiés (QSE, Qualified Scientists and Engineers). Par exemple, une étude récente sur les interactions des entreprises de la région du Sussex avec les universités de cycles supérieurs a montré que ces interactions augmentent avec l'emploi d'ingénieurs et de chercheurs qualifiés, ainsi qu'avec la taille des entreprises. Un aperçu de ces résultats est donné dans une étude sur les sources de connaissances utilisées pour résoudre des problèmes techniques dans le processus d'innovation au niveau de divers secteurs industriels. Elle a montré qu'il y a, dans leur façon d'utiliser les sources externes, une différence significative entre le personnel universitaire et celui formé dans l'industrie, tous deux compétents pour résoudre des problèmes.

Les personnes ayant reçu une éducation industrielle comptent plus sur celle-ci et leurs expériences pour résoudre des problèmes techniques, alors que celles ayant une formation universitaire l'utilisent pour élargir leurs ressources.

Gibbons et Johnston ont montré que les universitaires, compétents pour résoudre les problèmes, puisent fréquemment des informations dans la documentation scientifique et utilisent des contacts directs personnels avec les scientifiques dans le secteur de la recherche publique. En comparaison, les personnes formées dans l'industrie, également compétentes pour résoudre des problèmes, ont tendance à ignorer cette ressource. Ces constatations ont deux explications : premièrement, la personne qui détient une formation universitaire a acquis «la connaissance de la connaissance», c'est-à-dire la capacité de reconnaître quand des ressources internes disponibles sont inadéquates pour résoudre des problèmes particuliers et la capacité de savoir où et comment trouver l'information nécessaire. Deuxièmement, ils suggèrent que les personnes formées en entreprises, compétentes pour résoudre les problèmes, représentent un frein évident pour l'utilisation des sources scientifiques, inhibant ainsi le transfert de connaissances scientifiques vers des applications industrielles. Cependant, une fois cette barrière surmontée, les 
personnes compétentes pour résoudre des problèmes utilisent fréquemment et efficacement les sources scientifiques. En effet, l'apport fondamental de mécanismes tel le projet d'une société de formation pour les PME est de souligner la valeur de la R-D et de même que celle des relations industriesuniversités. De façon similaire, le programme en technologie avancée d'information «ALVEY » a démontré à plusieurs entreprises la valeur de la collaboration avec les universités.

Les associations de recherche coopérative ont été créées initialement pour assister des entreprises de taille plus petites dans les secteurs industriels lorsque ces dernières étaient incapables d'assumer les coûts d'une R-D interne. Cependant, on a découvert que les grandes entreprises ont plus de contacts avec des associations techniques et de recherche que les PME. Dans une certaine mesure, cela est lié au fait que les grandes entreprises disposent du temps et du personnel nécessaires pour établir de tels contacts.

Une autre étude a montré que les laboratoires internes et les organisations de recherche sous contrat sont complémentaires et, par conséquent, les associations de recherche coopérative ne peuvent pas pallier le manque de recherche interne aux entreprises. Une étude du soutien gouvernemental au sujet de la R-D industrielle en Norvège met en évidence les problèmes rencontrés par les petites entreprises sans ingénieurs ou chercheurs qualifiés qui essaient d'avoir accès aux compétences disponibles à l'intérieur des instituts de recherche. D'un côté, les PME éprouvent des difficultés à spécifier leurs besoins d'une manière technique, comme l'exigent les instituts de recherche. De l'autre, celles qui réussissent à surmonter cet obstacle seules donnent suite aux résultats de projets entrepris pour elles-mêmes par les instituts. Le rapport propose des recommandations pour dépasser ces problèmes. Il suggère que les projets ne devraient pas se terminer sans rapports écrits, mais qu'à la suite de ceux-ci, le personnel de l'institut pourrait consacrer du temps dans l'entreprise, mettant en œuvre les résultats. Il suggère aussi qu'un soutien financier soit apporté pour stimuler les entreprises à envoyer leur personnel dans les instituts dès le commencement d'un projet afin d'aider à définir exactement la prestation dont l'entreprise a besoin.

Ce résultat démontre bien que le manque d'ingénieurs et de chercheurs inhibe la croissance et l'innovation dans les PME. C'est pour cette raison que le gouvernement ouest-allemand a introduit un projet en 1979 pour subventionner les dépenses occasionnées par les emplois de R-D dans les PME.

L'étude du SPRU sur les relations extérieures des PME innovatrices a cependant montré qu'une proportion relativement élevée d'entreprises manquant d'ingénieurs et de chercheurs qualifiés est capable d'utiliser les projets du ministère du Commerce et de l'Industrie avec succès. Dans la 
majorité des cas, cela implique le recours à des consultants pour transférer des technologies ayant fait leur preuve mais que les entreprises ne peuvent assimiler aisément. Le même groupe d'entreprises a également réussi à faire usage des services des associations de recherche. On suggère que de tels services peuvent avoir eu un rapport avec l'industrie en général (problèmes reliés aux procédés, aux normes, aux essais et à la certification), plutôt qu'avec le développement de nouveaux produits exigeant des ressources techniques internes complémentaires.

De plus, après avoir entrepris une critique de la documentation en général et conduit des discussions avec des spécialistes de PME étrangères et britanniques, Rothwell et Beesley (1989) étaient :

[...] incapables de trouver une preuve attestant que le manque d'accès à la technologie et au savoir-faire constitue un frein considérable à la croissance dans les PME qui possèdent ce potentiel de croissance [...]

Les facteurs les plus importants pour déterminer la tendance naturelle d'une PME et sa capacité à avoir accès à des sources de technologies externes sont internes à cette entreprise. On peut, notamment et principalement, citer l'emploi d'ingénieurs et de chercheurs qualifiés et l'ouverture vers l'extérieur de la part de la direction.

\section{Relations PME et institutions de recherche fondamentale: une étude de cas}

Cette partie présente le contenu de récentes études sectorielles sur les relations PME et recherche fondamentale.

Elles couvrent trois types de PME:

- celles des secteurs traditionnels qui manquent d'ingénieurs et de chercheurs qualifiés ;

- celles employant du personnel scientifique et faisant usage de technologies établies, mais suffisamment intéressées pour se diversifier par le recours à de nouvelles technologies ;

- celles de haute technologie.

Ces études diffèrent légèrement de la typologie de Rothwell. Ses quatre catégories sont utiles dans un sens général, mais ne dévoilent pas complètement les comportements de rapprochement en recherche fondamentale, révélés dans les études sectorielles et technologiques de cas détaillées et présentées ci-dessous, alors que la relation avec la recherche fondamentale 
paraît très probable lors de l'intégration d'une technologie nouvelle. Ces études montrent malgré tout que les sources de connaissances externes évoluent aussi selon le degré de nouveauté de la technologie dans une entreprise.

\subsection{Les PME des secteurs traditionnels}

Cette partie contient des informations au sujet des PME de l'industrie agroalimentaire et de l'industrie de transformation du plastique. Rothwell soutient que les petites entreprises de ce secteur sont supposées utiliser la technologie produite par leurs fournisseurs de matériels et d'équipements. L'étude de cas suivante montre que les petites industries agro-alimentaires sont également assistées par les grandes entreprises de produits alimentaires qui agissent, au besoin, en tant qu'intermédiaires entre la recherche fondamentale et leurs petits fournisseurs. Il peut y avoir une relation équivalente entre les détaillants de vêtements et leurs fournisseurs. Les éléments un peu éclatés, présentés ici, semblent cependant suggérer que les détaillants recourent maintenant davantage à de gros fournisseurs en raison des dépenses élevées pour introduire de nouvelles technologies dans ce secteur. Nous constatons, à propos du secteur des fabricants de plastique, que le manque d'ingénieurs, de chercheurs qualifiés et de techniciens limite leur capacité à utiliser l'information technique disponible auprès de leurs fournisseurs et affecte la qualité de leurs produits.

\subsubsection{Les industries agro-alimentaires}

Une étude récente a montré que la majorité des détaillants en alimentation étaient responsables de l'introduction de produits innovants sur le marché en collaboration avec les fournisseurs. Une grande partie de ces innovations, influencées par les détaillants, sont apparues dans les produits frais, un domaine traditionnellement négligé par les fabricants d'épicerie emballée. Les détaillants alimentaires capables de jouer ce rôle sont ceux qui ont créé et qui construisent des services performants de technologies alimentaires, qui disposent d'une grande capacité dans la préparation des aliments, d'emballage, de stockage et de distribution. Ils ne développent pas seulement de nouveaux produits portant leurs propres labels, mais ils donnent aussi à leurs fournisseurs les instructions à suivre. Ces dernières englobent la qualité et le type d'ingrédients bruts jusqu'à la température du véhicule de livraison dans lequel les marchandises sont transportées. Les fournisseurs privilégiés sont les petites entreprises tant qu'elles peuvent fournir l'approvisionnement en volume nécessaire. 
Les technologues alimentaires des détaillants entretiennent des contacts très étroits avec les fournisseurs en passant une grande partie de leur temps dans les entreprises et en fournissant une assistance technique et analytique si nécessaire. Certains problèmes identifiés ne sont pas du ressort des technologues. Dans ce cas, ils agissent comme intermédiaires et recherchent les informations technologiques appropriées dans la documentation technique, auprès des associations de recherche, des instituts de recherche ou scientifiques gouvernementaux. Les détaillants transfèrent la connaissance technique à leurs fournisseurs afin de résoudre les problèmes qui ont causé de nombreux soucis, mais ils comptent aussi sur une coopération rapprochée entre les fabricants prêts à réaliser l'investissement nécessaire en équipement ou à modifier les processus de production. Des visites d'entreprises rapprochées fournissent également aux technologues alimentaires de détaillants une base pour évaluer la performance des installations disponibles. Ils peuvent, par conséquent, conseiller leurs fournisseurs sur la sélection et le développement des installations de transformation. Le partenariat entre les détaillants en alimentation et leurs fournisseurs s'est révélé efficace pour augmenter les normes d'hygiène et de qualité dans les PME, pour stimuler et satisfaire les besoins du client.

\subsubsection{Textiles et habillement}

En ce qui concerne les textiles et les vêtements, Marc \& Spencer a été un pionnier dans le transfert des progrès techniques vers une grande partie de ses petits fournisseurs. La poursuite de la recherche a permis d'identifier des modèles similaires dans d'autres grands magasins détaillants (comme BHS et C \& A). Le conseil technologique aux fournisseurs est assez répandu et fait partie des relations de sous-traitance au Royaume-Uni. Cependant, on a aussi la preuve que depuis 1980, Marc \& Spencer a opéré un glissement sensible vers des fournisseurs plus importants. Ce mouvement est relié au coût élevé des nouvelles technologies et à l'économie d'échelle requise pour leur rentabilité.

\subsubsection{La transformation du plastique}

Deux études sur l'industrie de la transformation du plastique, menées à environ 10 ans d'intervalle, montrent peu de changement dans la tendance actuelle des PME transformatrices de plastique à employer des ingénieurs et des chercheurs qualifiés. Ces deux études visaient à évaluer les besoins en personnel qualifié dans cette industrie. La première étude a traité de l'industrie en général et la deuxième s'est concentrée sur les polymères et les composites avancés. 
L'étude la plus récente a été réalisée à partir d'interviews approfondis menés auprès de 40 entreprises, parmi lesquelles un petit nombre seulement dépassait les 500 employés. Cette étude a mis en évidence l'idée que le changement technique était déterminé par le besoin de connaissances théoriques sur les caractéristiques des matériaux, sur leur comportement lors de leur transformation et sur leur performance. La connaissance théorique au sujet des matériaux et de leurs limites est utile pour concevoir de nouveaux produits et pour mettre au point des procédés appropriés pour tester matériaux et produits. Des connaissances en théorie de la régulation et en électronique sont utiles pour automatiser la conception et la production.

La majorité des transformateurs de plastique sont des PME. L'étude a montré qu'aucune des entreprises employant moins de 100 personnes ne disposait d'installations de laboratoire. Certaines de ces petites entreprises utilisent les services de consultants extérieurs pour le contrôle de la qualité, car elles ne disposent pas des équipements internes nécessaires. Le contrôle de la qualité est vu dans bon nombre d'entreprises comme une fonction de contrôle plutôt que de production, car aucun test de matériaux n'assure qu'ils répondent aux exigences requises. La plupart des entreprises ont cependant obtenu assistance et information auprès de services extérieurs et 26 des entreprises de l'étude ont fait appel à des établissements d'enseignement supérieur. Les différentes formes d'aide obtenue allaient de l'envoi de membre du personnel pour participer à des séminaires jusqu'à l'emploi d'universitaires en tant que consultants, en passant par l'obtention d'informations provenant de la recherche universitaire. Pour ce qui est du recrutement, seules les grandes entreprises embauchaient des diplômés. Les mouleurs de plastique considéraient les fournisseurs de poudre comme une source de compétences techniques. Cependant, les fabricants de poudre, tout en reconnaissant que la connaissance des mouleurs au sujet des polymères s'était accrue, pensaient qu'elle n'était toujours pas appropriée, spécialement dans les petites entreprises: non seulement ils n'avaient pas une compréhension appropriée des matériaux mais, en plus, ils avaient échoué dans l'utilisation des informations techniques sur les performances et la transformation des matériaux que les fabricants de polymères leur offraient. Plutôt que de recruter des techniciens ou des scientifiques capables de prévoir et d'éviter les ennuis, l'attitude prédominante dans l'industrie était de recourir à des experts dépanneurs [troubleshooters] pour faire face aux problèmes quand ils surgissaient.

L'étude la plus récente sur les composites et les polymères avancés notait que les moulistes de toute taille étaient engagés aussi bien dans l'ingénierie du moulage des polymères que dans les plastiques les moins avancés. Les utilisateurs finaux de ces nouveaux matériaux critiquaient le faible niveau théorique des entreprises de moulage, ainsi que leur manque d'expertise. Ces 
dernières ne pouvaient pas utiliser un contrôle statistique sur un équipement très complexe par manque de connaissances fondamentales du traitement. En effet, l'étude a montré que plusieurs entreprises en moulage n'employaient pas de diplômés d'universités, et seule une minorité en employait plus d'un. Les utilisateurs finaux étaient de ce fait très critiques quant à la qualité des produits des mouleurs. C'est pour cette raison qu'une société japonaise s'approvisionne en Allemagne.

Il y avait de toute évidence des liens entre les PME et le secteur privé et public de la recherche, bien que les PME eussent tendance à voir ces institutions de recherche comme éloignées des universitaires et déconnectées des réalités de la production.

\subsection{L'utilisation de nouvelles technologies}

Cette section a pour objet d'établir un rapport entre les entreprises qui emploient des ingénieurs et des chercheurs qualifiés et celles qui se diversifient à travers de nouvelles technologies. Bien qu'il y ait peu d'études détaillées sur ce sujet, quelques informations provenant d'autres études sont disponibles. Elles incluent l'attitude de spécialistes tels que les producteurs d'arômes pour l'agro-alimentaire et ceux du secteur audio haute fidélité.

\subsubsection{Les biotechnologies}

Une étude récente fut menée sur les projets de transferts technologiques vers les PME spécialisées en biotechnologies. Deux entreprises du secteur agroalimentaire étaient intéressées par l'exploitation du potentiel des biotechnologies. Les sociétés concernées s'impliquaient dans la R-D interne et avaient des liens avec les formations de leurs métiers traditionnels. Cependant, le manque d'ingénieurs et de chercheurs qualifiés internes spécialistes des expertises biotechnologiques faisait que les sociétés ne savaient pas où trouver les experts extérieurs appropriés, ni évaluer le potentiel des recherches annoncées dans la presse. Le manque de budget pour financer les recherches externes constituait une autre contrainte pour ses sociétés.

\subsubsection{L'équipement audio haute fidélité}

Le secteur audio est dominé par les PME qui tendent à produire une gamme limitée de produits. Une étude récente sur 61 entreprises a montré que $95 \%$ de celles-ci avaient créé moins de 200 produits, dont $50 \%$ entre 1 et 10 . Au début des années 70 , le marché de l'équipement de haute fidélité était stable 
et technologiquement stagnant. Le développement consécutif de nouvelles technologies audio a orienté les PME marginales vers les nouveaux créneaux de la haute fidélité. L'avantage majeur de ces sociétés sur leurs concurrents externes était leur flexibilité innée. Cet avantage est maintenant dépassé par l'introduction de nouvelles technologies produites par leurs nombreux concurrents de plus en plus flexibles de même que par le développement de nouvelles technologies audio telles que le DC (disque compact) et le R-DAT (technologie audionumérique). Les PME doivent profiter de l'accès aux nouvelles technologies audio si elles veulent rester compétitives. Les dépenses de R-D des entreprises varient de $4 \%$ du chiffre d'affaires pour les PME à $6,5 \%$ pour celles dont les gammes comprennent de 50 à 499 produits. Quelques entreprises ont une bonne utilisation des consultants externes. En effet, les PME utilisent ce champ pour accéder à la technologie du disque compact (DC) pendant que les grandes entreprises visent plutôt la recherche de conseil en design. De plus, ces entreprises audio forment des alliances avec de grandes sociétés pour obtenir des éléments et des technologies sophistiquées ; par exemple, plusieurs entreprises s'orientent vers la production de DC, ce qui nécessite l'accès à la technologie du laser, ce qui les a amenées à conclure des accords avec Philips. La moitié des entreprises de l'étude avaient contracté différents types de liens avec les établissements d'enseignement supérieur, mais l'étude ne renseignait pas sur l'utilisation de ces liens.

\subsection{Les PME de haute technologie}

Cette section rend compte de deux études sur les entreprises faisant de la recherche appliquée et sur celles exploitant les technologies émergentes. La première étude examinait les interactions des entreprises avec le secteur public de recherche dans trois champs technologique avancés : les biotechnologies, l'ingénierie des céramiques et les réseaux informatiques. Des entretiens avec les entreprises, on retire que seules des PME étaient engagées dans les deux dernières technologies citées. Toutes les entreprises de réseaux informatiques étaient des PME, comme l'étaient un grand nombre de fabricants de céramiques. Dans la section ci-après traitant de l'ingénierie avancée en céramique, l'information concernait aussi bien les PME que les filiales de grandes entreprises qui, dans la plupart des cas, opèrent dans des conditions similaires aux PME. L'information sur les liens de la recherche fondamentale avec les PME du secteur des biotechnologies est issue de l'étude déjà mentionnée. Cette section inclut aussi le secteur des instruments scientifiques, lequel possède de nombreuses similitudes avec les réseaux informatiques. 


\subsubsection{Ingénierie en céramique}

L'étude sur les entreprises exploitant cette technologie porte sur des entreprises de toutes tailles. Elle inclut les fournisseurs de matériels, des fabricants et des consommateurs finaux. Seules les PME étaient des entreprises de fabrication. Il est important de bien saisir les résultats relatifs aux PME en les resituant par rapport à leurs représentations propres. Les entreprises croient toutes que la faible implication du système universitaire dans les céramiques crée une réelle barrière quant à l'accès à la recherche fondamentale dans ce champ technologique. Mais les universités manquent souvent de connaissances appropriées ou d'équipements modernes, plus spécialement en production, pour fournir le type d'aide dont quelques sociétés auraient besoin. Le manque de moyens financiers dans les différents départements universitaires et la négligence des aspects de l'ingénierie dans les céramiques affectent l'utilisation de leurs compétences. De plus, les entreprises croient que leurs activités internes constituent la plus forte contribution à l'innovation, leur relation avec des sources extérieures provenant plus d'autres entreprises que de la recherche du secteur public.

On comptait deux PME parmi les fabricants en céramique impliqués dans cette étude. Notons que celles-ci possédaient une expérience des relations avec le secteur de la recherche fondamentale similaire à celle de deux petites entreprises de céramique, filiales de grandes sociétés. Ces dernières ont donc été prises en compte dans cette section. On peut remarquer que toutes ont dû faire face à des contraintes considérables de main-d'œuvre et de ressources financières en ce qui concerne leurs liens avec le secteur de la recherche fondamentale. Elles étaient toutes membres du British Ceram, association de recherche industrielle, et utilisaient une large gamme de ses services pour essayer de nouveaux équipements de production ou des tests d'analyses. Toutes les entreprises prêtaient attention aux départements d'universités ayant des connaissances pertinentes et aux installations disponibles du Laboratoire national de physique de Harwell.

Trois de ces sociétés utilisaient des ingénieurs et des chercheurs qualifiés. De plus, elles travaillaient étroitement avec leurs clients pour mieux connaître les performances de leurs produits. Elles en ont aussi tiré un grand intérêt en raison de la participation du ministère du Commerce et de l'Industrie au niveau de ses programmes CARE «Ceramic Applications in Reciprocating Engines » [Application céramique dans les moteurs alternatifs] et ACT «Advanced Ceramics for Turbines» [Céramique avancée pour les turbines]. Ces trois entreprises ont toutes été impliquées dans les programmes européens, mais seulement une d'elles a participé au programme LINK. Une autre de ces sociétés était bien consciente de la nécessité de mettre en place 
des systèmes de formation, mais manquait de temps et de ressources pour instaurer son propre programme de formation.

Le manque de ressources limite les contacts établis entre entreprises et universités. Elles s'engagent dans des liens de collaboration peu coûteux, tels que les actions de conseil ou de parrainage auprès des étudiants participant au CASE. Cependant, elles passent la plupart de leur temps à chercher des personnes qualifiées et des études spécifiques, tout en enrichissant et en gardant leurs contacts existants. Ces contacts sont utilisés de manière informelle pour l'échange d'informations, la résolution de problèmes, l'apprentissage de nouvelles techniques expérimentales et l'accès aux instruments scientifiques universitaires. Un système de troc règle beaucoup de ces contacts informels. En retour, les entreprises échangent du matériel contre des informations et de l'aide des universités.

La quatrième société aimerait développer davantage ses contacts avec la recherche mais étant située à la périphérie du Royaume-Uni, dans une région qui manque de savoir-faire, les coûts et le temps nécessaires pour se rendre à ces centres d'expertises sont considérables. De ce fait, les contacts sont occasionnels. Cette société manque aussi d'ingénieurs et de chercheurs qualifiés, à part deux équipes qui ont reçu une année de formation en ingénierie.

Ces trente dernières années, cette entreprise n'a eu que des réussites avec deux des trois produits pour lesquels elle a acheté une licence, l'un des deux ayant pu bénéficier, au moment de son lancement, des recommandations et des conseils de la société américaine concédante et d'un expert universitaire. Les produits de haute technologie en céramique de cette entreprise sont utilisés dans plusieurs secteurs, équipements de radar et de laser, mais aussi par des industries pétrolières, chimiques, nucléaires et spatiales. Elle exporte dans le monde entier, dont $60 \%$ de sa production vers l'Allemagne. En comparaison avec les fabricants de plastiques déjà mentionné, vérifier l'évolution des matières premières est indispensable pour s'assurer d'avoir les bonnes spécifications. Le British Ceram mène des tests et des nouveaux lots de matériaux ne sont jamais utilisés jusqu'à ce que les résultats des tests confirment qu'ils correspondent aux spécifications. La direction de l'entreprise a été formée pour éviter toute défaillance dans le processus impliqué dans la production des céramiques.

Fait surprenant, cette société a bénéficié de l'initiative d'un enseignant ingénieur venant d'une université locale, qui aborda la société en lui demandant si elle était intéressée à se joindre à un projet concernant les techniques en céramique. Ils développèrent ensemble un projet financé par le SERC. La société apporta beaucoup d'aide en temps, en équipement, en savoir-faire 
et en matériel, tandis que le professeur passait une bonne partie de son temps à l'usine pour enseigner la céramique et les différents processus associés. Le directeur technique passait quant à lui une partie de son temps à l'université. Le résultat du projet fut un nouveau procédé, qui a déjà été utilisé par un client. Mais le plus important, c'est que l'entreprise demeure en contact avec l'université locale à cause de ses compétences en céramique. La société est même sur le point d'étendre géographiquement ses expertises en céramique grâce au partenariat établi avec un professeur d'une université située à proximité - toujours sur l'initiative universitaire.

\subsubsection{Les ordinateurs}

Dans ce secteur, les interviews ont été effectuées auprès des six entreprises qui représentent toutes les sociétés du Royaume-Uni fabriquant et commercialisant du matériel informatique de façon parallèle. Les principaux groupes de clients de ces sociétés sont les universités et les laboratoires de recherche de grandes sociétés, y compris ceux qui exécutent des contrats de la défense civile. Les universités ont souvent recours aux financements du gouvernement pour acquérir des équipements ; en effet, celles-ci cherchent une puissance de calcul pour résoudre les problèmes scientifiques (par exemple, en physique et en biochimie), mais aussi des personnes capables de comprendre le potentiel des réseaux informatiques. De plus, elles sont disposées à investir leur temps et leurs efforts dans l'apprentissage des différents systèmes de réseaux et à développer des logiciels propres à leur environnement. De façon similaire, quelques grandes sociétés dans l'aérospatiale et l'industrie pétrolière ont pris conscience du potentiel de l'informatique en parallèle : elles l'ont incorporé dans leurs systèmes pour une première phase d'essais afin de décider quels logiciels sont les plus appropriés à leurs besoins. Au niveau de la recherche, on tend à être tolérant en ce qui concerne les problèmes avec le matériel et les logiciels. Dans un sens, les premiers utilisateurs sont considérés comme «cobayes » pour tester et développer la technologie afin qu'elle soit acceptée comme produit fini pour de grands marchés potentiels tels que les institutions financières, les compagnies aériennes et d'autres services nécessitant la mise sur pied d'importants réseaux.

Toutes les sociétés pensent que le savoir scientifique et technologique nécessaires au développement de nouveaux produits est déjà disponible en interne ; en effet, le savoir universitaire est perçu comme restant derrière les avancées de l'industrie. Le travail de développement dans toutes les sociétés se concentre sur la conception (design industriel). Certains travaux sur des applications de logiciels sont traités en externe par d'autres sociétés et sont aussi le centre de quelques collaborations en recherche avec les universités. 
Cela s'explique par le fait que le développement des applications de logiciels nécessite un savoir portant non seulement sur les ordinateurs et la programmation, mais aussi sur le secteur pour lequel l'application est développée. Toutes les sociétés ont des liens avec la recherche universitaire, car elles sont intéressées par l'étude des applications pratiques que les universitaires mettent en œuvre. Les idées de nouvelles applications peuvent venir, soit en visualisant les travaux issus des universités, soit en identifiant les consommateurs pour un type de produit donné. Les institutions de recherche du secteur public sont les premières utilisatrices de ces systèmes, en fournissant des informations sur les marchés potentiels pour des réseaux informatiques, mais aussi parce qu'elles ont un rôle stratégique de consommation importante de ces développements.

\subsubsection{L'instrumentation scientifique}

Il paraît évident que les utilisateurs d'instruments scientifiques jouent un rôle important dans l'innovation de ce secteur. Une grande proportion d'utilisateurs innovants se trouve dans les laboratoires de recherche du secteur public. Cela est en partie explicable par le fait que les avancées radicales dans l'instrumentation scientifique sont souvent associées de près aux percées dans la science. En effet, il a été suggéré que le succès de l'innovation dans les instruments se présente à travers le «triangle d'or» des relations de la figure 1 .

L'inventeur-chercheur, en testant et opérationnalisant le prototype d'usine d'un instrument, agit comme utilisateur scientifique leader pour la société qui commercialise cet instrument. En retour, le chercheur est le premier à bénéficier des améliorations des performances techniques faites pendant le développement. L'implication de ces premiers utilisateurs venant de l'industrie ou du gouvernement permet d'assurer que les besoins du marché sont pris en compte pendant le processus d'innovation, et garantit ainsi des ventes initiales importantes. De plus, le statut de compétences techniques des premiers utilisateurs permet de promouvoir une rapide diffusion des nouveaux instruments. En effet, il y a de fortes similitudes entre les réseaux informatiques et les instruments scientifiques. À cet égard, M. Irvine a souligné que les programmes LINK les plus réussis à ce jour, en termes de consolidation de marché à terme, sont deux programmes axés sur l'instrumentation: les Systèmes de mesures industriels [IMS : Industrial Measurement Systems] et la Technologie pour les mesures analytiques et physiques [TAPM : Technology for Analytical and Physical Measurement]. Selon lui, cela pourrait provenir du fait que les programmes LINK fournissent les conditions optimales pour le succès de l'innovation en instrumentation. 
Le SERC finance justement les chercheurs universitaires dont l'objectif est d'améliorer les nouveaux instruments, pendant que les fonds provenant du ministère du Commerce et de l'Industrie permettent aux PME de s'engager dans des programmes de développement risqués de commercialisation d'instruments. Les premiers utilisateurs industriels et la majorité des fournisseurs sont aussi attirés par le programme pour les PME.

\section{FIGURE 1}

\section{Le triangle d'or des rapports et du succès de l'innovation dans l'instrumentation scientifique}

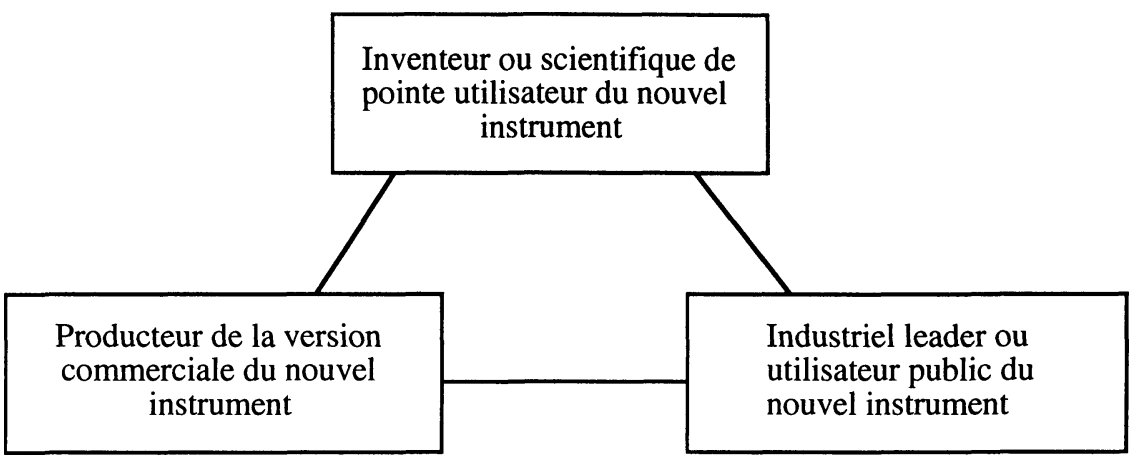

\subsubsection{Les biotechnologies}

Deux études qui examinent les relations externes des PME en biotechnologie ont été recensées. La première montre que la moitié des entreprises enquêtées utilisent régulièrement des relations techniques externes, dont la plus importante est nouée avec les universités. La seconde étude montre que la majorité des 39 PME interviewées exploitent activement les biotechnologies. Ces dernières sont impliquées dans un grand nombre de secteurs, incluant la pharmacie, les plantations, l'alimentation, la chimie, les usines de retraitements. Ces PME ont donc une grande diversité d'activités fondées sur les progrès scientifiques en biotechnologie.

La majorité des entreprises interviewées font de la recherche intensive. Elles s'impliquent fortement en recherche interne, mais font aussi beaucoup appel à la recherche externe. Les recherches interne et externe jouent un rôle différent dans la stratégie de la société : l'interne est largement orientée vers l'acquisition de procédés et de produits directement pour le marché, tandis que l'externe est orientée vers la découverte de concepts pour les générations futures de produits et de procédés. Les entreprises se servent des universitaires pour une gamme d'activités incluant: 
- des contrats directs pour la recherche scientifique et plus tournés vers les applications commerciales ;

- des consultations;

- des accords de licence;

- l'utilisation des équipements universitaires;

- l'évaluation de la performance du matériel de recherche que les entreprises produisent;

- l'adhésion au conseil scientifique de l'entreprise.

Bien que la plupart des contrats directs couvrent les coûts d'un ou deux chercheurs, certains ont dépassé ces coûts. Quelques directions de certaines sociétés apportent aussi leur contribution dans les activités universitaires, comme :

- l'adhésion aux comités de conseil en recherche;

- l'enseignement à l'université ;

- le parrainage d'étudiants de maîtrise en science;

- des aides pour les étudiants en formation professionnelle en alternance.

Cependant, les petites entreprises ne sont pas intéressées en général par la recherche à long terme. Elles subissent une pression interne de la part des actionnaires pour obtenir un retour rapide d'investissement sur la recherche qu'ils commanditent. Une période de trois ans, depuis l'élaboration jusqu'à la rentabilité du produit, constitue le cadre de temps nécessaire. Seul un flux régulier de recettes crée les conditions à partir desquelles les firmes commencent à penser à mettre en place un fonds de recherche externe. Malgré ces contraintes, un quart des entreprises déclarent que leurs liens avec la recherche universitaire sont primordiaux, bien que peu d'entre elles soient intéressées par des projets de recherche à long terme.

Pour la plupart des entreprises, la principale barrière à l'utilisation de la recherche externe est le manque de fonds. Dans un certain nombre de cas, le problème est causé par un manque d'argent ou de contacts avec des spécialistes extérieurs. Quelques PME interrogées ont affirmé que des secteurs de la recherche les intéressant étaient négligés. Ces griefs portent sur trois domaines: l'enzymologie de base, la physiologie microbienne et la modélisation des systèmes de culture de tissus. Il existe cependant quelques domaines de la biotechnologie où le petit nombre d'universités qui possèdent des connaissances de pointe, ainsi que les chercheurs concernés, sont entièrement 
engagés auprès des grandes sociétés. Bon nombre de PME étroitement liées aux grandes entreprises, ainsi qu' aux fournisseurs de produits intermédiaires, affirment qu'elles ont bénéficié de tels liens dans plusieurs domaines. Ils représentent une source utile de connaissances et d'idées pour de nouveaux produits.

Par contre, très peu de PME ont une compréhension claire des occasions de créer des liens avec la recherche fondamentale proposée par les projets publics. Elles croulent sous le volume de lettres envoyées par diverses organisations de biotechnologie et ont des difficultés à comprendre les différences entre les divers projets et les organisations qui en font la promotion. Les organisations qui connaissent les projets s'aperçoivent qu'elles ont été choisies pour répondre aux besoins des entreprises, mais la plupart des projets ne conviennent pas aux PME parce qu'ils impliquent du long terme, de la prérecherche compétitive ou requièrent une collaboration avec d'autres organisations sur les plans national et international, ce qui demande beaucoup trop de temps de mise en place pour les petites entreprises. Malgré ces critiques, un petit nombre d'entreprises ont été impliquées dans des projets de la CEE et environ un tiers des sociétés ont reçu des formulaires à remplir pour les bourses du ministère du Commerce et de l'Industrie. Le précédent plan d'encouragement à l'innovation est devenu plus en vogue. Un grand nombre de ces firmes éprouvent de la difficulté à recruter des chercheurs au niveau postdoctoral et à gérer le processus d'apprentissage interne des diplômés pour qu'ils acquièrent les qualifications requises. Ce n'est pas possible lorsqu'elles désirent recruter de nouveaux spécialistes, et plusieurs pensent que le programme des firmes enseignantes [Teaching Company Scheme] constituerait une solution idéale au problème.

\section{Conclusion}

La capacité des entreprises à transformer des idées en produits réussis du point de vue commercial et en procédés est un facteur clé de la croissance économique. Les PME contribuent de façon croissante à l'innovation en général; en particulier dans les secteurs des machines, des instruments, des véhicules, de la construction navale et du bâtiment. Depuis 1970, les petites entreprises du Royaume-Uni ont recouru de façon croissante aux connaissances externes. Mais les PME sont très hétérogènes et leurs besoins en savoir technique externe varient considérablement suivant les secteurs.

Les établissements d'enseignement supérieur assument deux volets d'activités : l'enseignement et la recherche. Ils collaborent avec l'industrie principalement par le biais de formation universitaire de troisième cycle, mais 
aussi par la publication des résultats de recherche. Au cours des années 80 , nous avons cependant été témoins d'une forte croissance des mécanismes de liens entre les universités et l'industrie.

Le ministère du Commerce et de l'Industrie a de nombreux projets pour promouvoir l'innovation au sein des entreprises, avec des objectifs spéciaux pour les PME. De plus en plus, la politique du SERC a été orientée vers un soutien croissant de la recherche à vocation industrielle. Les projets, comme le schéma de la firme enseignante [Teaching Company Scheme] et le programme ALVEY, peuvent avoir été utiles pour les PME dans l'évaluation de la R-D et de la collaboration avec l'université.

Le tableau 3 résume les principales sources d'informations techniques utilisées par les PME dans les différents secteurs considérés. Il montre que les PME dans la plupart des secteurs sont en contact avec la recherche fondamentale à travers une variété de sources externes, et il confirme les conclusions du Centre de recherche sur les petites entreprises (voir le tableau 2) en montrant que les entreprises ont la plupart du temps d'importantes sources externes scientifiques et d'informations techniques. Il indique aussi que les établissements d'enseignement supérieur ont une importance spécifique pour les PME de haute technologie. Les études de cas révèlent que les PME de haute technologie peuvent manquer de ressources financières pour mettre en place des contrats de recherche dans les universités, qu'elles utilisent souvent de simples liens informels ou des dispositifs d'échanges pour profiter des sources d'informations dont elles ont besoin. En revanche, les PME des secteurs traditionnels n'emploient pas les ressources des établissements d'enseignement supérieur. Cette situation et l'expérience de petites entreprises qui tentent de se diversifier dans les nouvelles technologies soulignent l'importance, pour les entreprises, d'employer des scientifiques et des ingénieurs qualifiés dans les disciplines appropriées si elles veulent exploiter la recherche fondamentale.

Les études de cas montrent que les besoins des PME en termes de recherche fondamentale, ainsi que les différents types de savoir requis, varient selon les secteurs et les technologies. Les PME recherchent une large variété d'intrants provenant de la recherche fondamentale: personnel à embaucher, conseil, utilisation d'instruments, tests analytiques, accès au savoir scientifique. Les politiques conçues pour soutenir les liens des PME avec la recherche du secteur public devraient prendre cette variété en compte. En effet, les projets réussis peuvent être apparentés à ceux qui coïncident fortuitement avec les besoins spécifiques des secteurs tels que les programmes CARE et ACT pour le secteur de la céramique, et les programmes IMS et TAPM LINK pour le secteur des instruments scientifiques. 
Les études de cas confirment le point de vue déjà exprimé, selon lequel la propension et la capacité des PME à accéder à la recherche fondamentale sont largement déterminées par l'emploi d'ingénieurs et de chercheurs qualifiés bien au fait des perceptions et des intérêts managériaux relatifs à la recherche externe et à la technologie. Le programme des entreprises enseignantes [Teaching Company Scheme] et le programme ALVEY paraissent avoir de très bons résultats quant aux avantages liés à l'emploi de scientifiques et aux contacts avec la recherche fondamentale.

TABLEAU 3

Les principales sources externes scientifiques et de savoir technologique des PME, par secteurs

\begin{tabular}{|c|c|c|c|c|}
\hline Secteurs & $\begin{array}{c}\text { Établissement } \\
\text { d'enseignement } \\
\text { supérieur }\end{array}$ & $\begin{array}{c}\text { Autres } \\
\text { entreprises }\end{array}$ & Consultants & $\begin{array}{c}\text { Associations } \\
\text { de } \\
\text { recherche }\end{array}$ \\
\hline $\begin{array}{l}\text { Traditionnels } \\
\text { Production alimentaire } \\
\text { Textile et vêtement } \\
\text { Plastiques }\end{array}$ & & $\begin{array}{l}* \\
*\end{array}$ & $*$ & \\
\hline $\begin{array}{l}\text { Utilisant de nouvelles } \\
\text { technologies } \\
\text { Ingrédients alimentaires } \\
\text { Équipements audio } \\
\text { haute fidélité }\end{array}$ & $*$ & $*$ & $*$ & \\
\hline $\begin{array}{l}\text { Technologies émergentes } \\
\text { Céramiques avancées } \\
\text { Réseaux informatiques } \\
\text { Instruments scientifiques } \\
\text { Biotechnologies }\end{array}$ & $\begin{array}{l}* \\
* \\
* \\
*\end{array}$ & $\begin{array}{l}* \\
* \\
* \\
*\end{array}$ & & $*$ \\
\hline
\end{tabular}

Un facteur important relativement aux firmes construites autour de nouvelles technologies est que le manque de recherche scientifique du secteur public ou d'expertise dans des domaines spécifiques peuvent agir comme un obstacle dans l'établissement de liens université-industrie. Cela est peut-être inévitable pour les entreprises qui développent des stratégies de niches dans des champs en expansion rapide comme les biotechnologies. Il existe cependant quelques domaines où les programmes de recherche publique pourraient être utiles à l'industrie, en construisant des spécialités pertinentes : génie enzymatique, système d'installation de culture de tissus, etc. L'écart est plus grand et plus significatif dans les matériaux externes reliés au savoir fondamental, au financement d'une instrumentation moderne dans les départements universitaires et au nombre de recrues ayant reçu une formation. 


\section{Bibliographie}

BRAHAM, P. (1985), « Marks \& Spencer : a technological approach to retailing», dans E. Rhodes et D. Wield (dir.), Implementing New Technologies, Choice, Decision and Change in Manufacturing, Oxford, Basil Blackwell.

DEPARTMENT OF TRADE AND INDUSTRY AND CENTRAL OFFICE OF INFORMATION (1992), Technology and Change. Help for Business, 2e édition, Londres, HMSO.

FAUlKner, W., J. SenKer et L. Velho (1995), Knowledge Frontiers : Public Sector Research and Industrial Innovation in Biotechnology, Engineering Ceramics and Parallele Computing, Oxford, Oxford University Press.

GibBONS, M. et R. JOHNSTON (1974), «The roles of science in technological innovation », Research Policy, vol. 3, n 3, p. 221-242.

GuY, K. et L. GeORGHiou (1991), Evaluation of the Alvey Programme for Advanced Information Technology, Londres, HMSO.

IRVINE, J. (1991), «Government policies for promoting innovation in scientific instruments : some lessons from recent overseas experience », Innovation in the Australian Scientific and Medical Instruments Industry, Canberra, Australian Government Publishing Service.

IRVINE, J., B. MARTIN et M. SCHWARZ en collaboration avec K. PAVITT et R. ROTHWELL (1987), «Government support for industrial research in Norway: a SPRU report », Centre de recherche sur les politiques scientifiques, Brighton, Sussex University.

Lowe, S. et R. Rothwell (1987), «The Sussex technology transfer centre. A background report », Centre de recherche sur les politiques scientifiques, Brighton, Sussex University.

MILNE, S. (1991), «Small firms, industrial reorganisation, and space : the case of the UK high-fidelity audio sector », Environment and Planning A, vol. 23, p. 833-852.

Monck, C.S.P., P. Quintas, P. WynarczyK, R. Porter et D. Storey (1988), Science Parks and the Growth of High Technology Firms, Londres, HMSO.

MOWERY, D. (1982), The Relationship Between Intrafirm and Contractual Forms of Industrial Research, 1900-1940, Graduate School of Business Administration, Harvard University.

OAKEY, R., R. ROTHWELl et S. COOPER (1988), Management of Innovation in High Technology Small Firms, Londres, Pinter Publishers.

OAkey, R., W. Faulkner, S. CoOper et V. Walsh (1990), New Firms in the Biotechnology Industry, Londres, Pinter Publishers.

PAVITT, K., M. RoBSON et J. TOWNSEND (1987), « The size distribution of innovating firms in the UK: 1945-1983», The Journal of Industrial Economics, vol. 35, $\mathrm{n}^{\circ} 3$, p. 297-316. 
Rigg, M., I. Christie et M. White (1989), Advanced Polymers and Composites: Creating the Key Skills, Rapport préparé pour Skills Unit of the Training Agency, Policy Studies Institute, Sheffield, Training Agency.

ROTHWELL, R. (1975), «Information patterns in innovation» dans A. Robertson et R. Rothwell (dir), Scientific and Technical Information Needs in $R-D$ Laboratories, Centre de recherche sur les politiques scientifiques, Brighton, Sussex University.

ROTHWELL, R. (1990), «External networking and innovation in small and medium sized manufacturing firms in Europe », Communication présentée à Network of Innovators Workshop, 1-3 mai, Montréal, Canada et Centre de recherche sur les politiques scientifiques, Brighton, Sussex University.

Rothwell, R. et M. BeEsley (1987), «ACARD barriers to growth study. The importance of technology transfer », Communication présentée à ACARD, Centre de recherche sur les politiques scientifiques, Brighton, Sussex University.

ROTHWELL, R. et M. BEESLEY (1989), «The importance of technology transfer » dans J. Barber, S. Metcalfe et M. Porteous (dir.), Barriers to Growth in Small Firms, Londres, Routledge.

SENKER, J. (1988), A Taste for Innovation: British Supermarkets' Influence on Food Manufacturers, Bradford, Horton Publishing Ltd.

SENKER, J. (1991), «The biotechnology directorate's programmes for technology transfer in relation to small and medium sized firms », Centre de recherche sur les politiques scientifiques, Brighton, Sussex University.

SENKER, J. (1992), «Informal contacts between academic and industrial research : a key to effective technology transfer », Communication présentée à Technology Transfer and Implementation, Compte rendu de la conférence TTI, Faringdon, Teaching Company Directorate.

SENKER, J.M. et W. FAULKNER (1992), «Industrial use of public sector research in advanced technologies : a comparison of biotechnology and ceramics' », $R-D$ Management, avril, p. 157-175.

SHAw, B. (1985), "The role of interaction between the user and the manufacturer in medical equipment innovation », $R-D$ Management, vol. $15, \mathrm{n}^{\circ} 4$, p. 283-93.

Small Business Research Centre (1992), The State of British Enterprise, Cambridge, Department of Applied Economics, Cambridge University.

TILER, C. et M. GIBBONS (1991), «A case study of organizational learning: the UK Teaching Company Scheme», Industry and Higher Education, mars, p. 47-55.

Walsh, V., J. Townsend, P. Senker et C. HuggetT (1980), « Technical change and skilled manpower needs in the plastics processing industry », Centre de recherche sur les politiques scientifiques, Brighton, Sussex University. 